\title{
Infrared Emission from Kilonovae: The Case of the Nearby Short Hard Burst GRB 160821B
}

\author{
Mansi M. Kasliwal ${ }^{1}$, Oleg Korobkin ${ }^{2}$, Ryan M. Lau ${ }^{1}$, Ryan Wollaeger ${ }^{2}$, and Christopher L. Fryer ${ }^{2}$ \\ ${ }^{1}$ Division of Physics, Mathematics and Astronomy, California Institute of Technology, Pasadena, CA 91125, USA \\ ${ }^{2}$ Computational Methods Group (CCS-2), Los Alamos National Laboratory, P.O. Box 1663, Los Alamos, NM, 87545, USA \\ Received 2017 May 5; revised 2017 June 13; accepted 2017 June 13; published 2017 July 12
}

\begin{abstract}
We present constraints on Ks-band emission from one of the nearest short hard gamma-ray bursts, GRB 160821B, at $z=0.16$, at three epochs. We detect a red relativistic afterglow from the jetted emission in the first epoch but do not detect any excess kilonova emission in the second two epochs. We compare upper limits obtained with Keck I/ MOSFIRE to multi-dimensional radiative transfer models of kilonovae, that employ composition-dependent nuclear heating and LTE opacities of heavy elements. We discuss eight models that combine toroidal dynamical ejecta and two types of wind and one model with dynamical ejecta only. We also discuss simple, empirical scaling laws of predicted emission as a function of ejecta mass and ejecta velocity. Our limits for GRB 160821B constrain the ejecta mass to be lower than $0.03 M_{\odot}$ for velocities greater than $0.1 \mathrm{c}$. At the distance sensitivity range of advanced LIGO, similar ground-based observations would be sufficiently sensitive to the full range of predicted model emission including models with only dynamical ejecta. The color evolution of these models shows that $I-K$ color spans 7-16 mag, which suggests that even relatively shallow infrared searches for kilonovae could be as constraining as optical searches.
\end{abstract}

Key words: gamma-ray burst: individual (GRB 160821B, GRB 130603B) - gravitational waves - nuclear reactions, nucleosynthesis, abundances - stars: black holes - stars: neutron

\section{Introduction}

Short hard bursts (SHBs) of $\gamma$-ray emission are purportedly neutron star mergers where the jet is conveniently pointed toward us (see Berger 2014 for a review). The same violent mergers are promising sources of gravitational-wave emission in the advanced LIGO frequency band, albeit independent of orientation and limited to the local universe.

The discovery of afterglows of SHBs has been much more challenging than that of long soft bursts due to their intrinsic faintness. In addition to an afterglow, long bursts have been shown to be accompanied by a broad-line Type Ic supernova (e.g., Galama et al. 1998). One may also expect "kilonova" emission in SHBs from radioactive decay of heavy elements synthesized in these extreme environments (see Fernández \& Metzger 2016 for a review). Kilonova emission could be red due to the opacities of heavy element lines (Barnes \& Kasen 2013; Kasen et al. 2013). Possible excess emission has also been reported for two nearby short bursts: excess optical emission was seen in GRB 080503 (albeit with a less secure redshift; Perley et al. 2009) and infrared emission in GRB 130603B (Tanvir et al. 2013; Fong et al. 2014). But the accompanying excess X-ray emission in both GRB 080503 and GRB $130603 \mathrm{~B}$ cannot be easily explained by kilonova models. Additional claims of excess emission include GRB 050709 (Jin et al. 2016) and GRB 060614 (Jin et al. 2015). Constraining upper limits on excess emission include GRB 150101B (Fong et al. 2016).

The discovery of GRB 160821B (Siegel et al. 2016) by the Swift satellite (Gehrels et al. 2004), one of the nearest SHBs, presented another opportunity to look for any excess emission hinting at heavy element radioactivity. An optical afterglow (Xu et al. 2016) and radio afterglow (Fong et al. 2016) were detected. A spectrum was obtained suggesting a very low redshift of 0.16 (Levan et al. 2016). Deep HST follow-up observations were also undertaken (Troja et al. 2016; E. Troja et al. 2017, in preparation). In this Letter, we present a search for excess infrared emission at the $\mathrm{Ks}$ band with Keck I/MOSFIRE.

\section{Observations}

We obtained three epochs of deep imaging of GRB 160821B with the MOSFIRE (McLean et al. 2012) instrument mounted on the Keck I telescope (Table 1) at 4.3 days, 7.5 days, and 8.4 days after the $\gamma$-ray burst. Data were taken in correlated double sampling mode with an integration time of $4.4 \mathrm{~s}$ and 7 co-adds in each exposure. Multiple well-dithered exposures were stacked on each night: specifically, 45 frames, 29 frames, and 25 frames were stacked for the three epochs. Data were reduced using standard procedures and calibrated relative to the 2MASS survey (Skrutskie et al. 2006). We marginally detect the afterglow in the first epoch (at $3 \sigma$ ) and report limiting magnitudes at the position of the afterglow of GRB 160821B for the second two epochs. All data are summarized in Table 1.

\section{Models}

A wide variety of models exist in the literature that predict different kilonova signatures based on different opacity assumptions (e.g., Barnes et al. 2016; Rosswog et al. 2016). Here, we focus on a detailed comparison of our observations to only one family of models with the reddest opacity predictions (model details are described in Wollaeger et al. 2017). This family of nine models uses multi-dimensional radiative transfer simulations with a new treatment of multigroup opacity broadening, an approach described in Fontes et al. (2017). They are based on dynamical ejecta morphologies (Rosswog et al. 2014), computed by long-term evolutions with radioactive heating source, following simulations of neutron star mergers in Rosswog (2013). Nucleosynthesis and radioactive heating 
Table 1

Ks-band Data on GRB 160821B

\begin{tabular}{llllll}
\hline \hline MJD (Phase) & Instrument & Filter & Apparent mag (Vega) & Apparent mag (AB) & Absolute Mag (AB) \\
\hline $57626.234(+4.3$ days) & Keck I/MOSFIRE & Ks & $22.19_{-0.31}^{+0.44}$ & $24.04_{-0.31}^{+0.44}$ & -15.4 \\
$57629.402(+7.5$ days $)$ & Keck I/MOSFIRE & Ks & $>22.17(3 \sigma)$ & $>24.02(3 \sigma)$ & -15.4 \\
$57630.321(+8.4$ days $)$ & Keck I/MOSFIRE & Ks & $>22.0(3 \sigma)$ & $>23.85(3 \sigma)$ & -15.6 \\
\hline
\end{tabular}

are computed using nuclear network code WinNET (Korobkin et al. 2012; Winteler 2014) that is derived from the BasNet network (Thielemann et al. 2011). Reaction rates for nucleosynthesis are taken from the Rauscher \& Thielemann (2000) compilation for the finite range droplet model (FRDM; Möller et al. 1995), including density-dependent weak reaction rates (Arcones \& Martínez-Pinedo 2011) and fission (Panov et al. 2010, 2005). We calculate radioactive heating energy partitioning between different decay species and their thermalization, using empirical formulae derived in Barnes et al. (2016). Radiative transfer simulations are performed using the open source code SuperNu ${ }^{3}$ (Wollaeger \& van Rossum 2014), which implements a 3D semi-implicit multigroup Monte Carlo solver.

The simplest model, dubbed SAd, features spherically symmetric analytic density distribution and contains dynamical ejecta only, with corresponding heating rates and opacities. This model is intended as the "worst-case" scenario of a merger in which the brighter wind component is not present, or is obscured by the so-called "lanthanide-curtain" (Kasen et al. 2015), and also because this model is the least luminous due to its spherical shape with the lowest surface area. Thus, the SAd model predicts the faintest kilonova signal.

The other eight models combine four different morphologies of dynamical ejecta (A-D from Table 1 in Rosswog et al. 2014) with two spherically symmetric analytic models of wind, "wind 1" and "wind 2." The wind carries two different nucleosynthetic compositions from representative tracers $\mathrm{H} 5$ and $\mathrm{H} 1$ in Perego et al. 2014, respectively. These models are abbreviated as $\gamma A_{1}, \gamma B_{1}, \gamma C_{1}, \gamma D_{1}, \gamma A_{2}, \gamma B_{2}, \gamma C_{2}$, and $\gamma D_{2}$.

Crude empirical scaling laws for the peak magnitudes ( $\mathrm{mp}$ ) in JHKs bands, depending on masses $(m)$ and velocities $(v)$, are as follows (for more details, see Wollaeger et al. 2017):

$$
\begin{aligned}
& m p_{J}=m p_{J 0}-0.93 \log \left(m / m_{0}\right)-1.61 \log \left(v / v_{0}\right), \\
& m p_{H}=m p_{H 0}-0.95 \log \left(m / m_{0}\right)-1.55 \log \left(v / v_{0}\right), \\
& m p_{K}=m p_{K 0}-0.99 \log \left(m / m_{0}\right)-1.53 \log \left(v / v_{0}\right) .
\end{aligned}
$$

With these scaling formulas, starting with peak JHKs magnitudes $m p_{J 0}, m p_{H 0}, m p_{K 0}$ for a model with some mass $\mathrm{m}_{0}$ and some velocity $\mathrm{v}_{0}$, we can estimate the peak magnitudes for a different mass and velocity. Furthermore, we note that the time to maximum light also scales with ejecta mass and median velocity (cf. Grossman et al. 2014):

$$
t_{p} / t_{p 0}=\left(m / m_{0}\right)^{0.32}\left(v / v_{0}\right)^{-0.60} .
$$

These empirical fits are obtained using gray opacity models. The above models do not assume gray opacity, and hence we caution that these empirical fits are only an approximation.

\footnotetext{
3 https://bitbucket.org/drrossum/supernu/wiki/Home
}

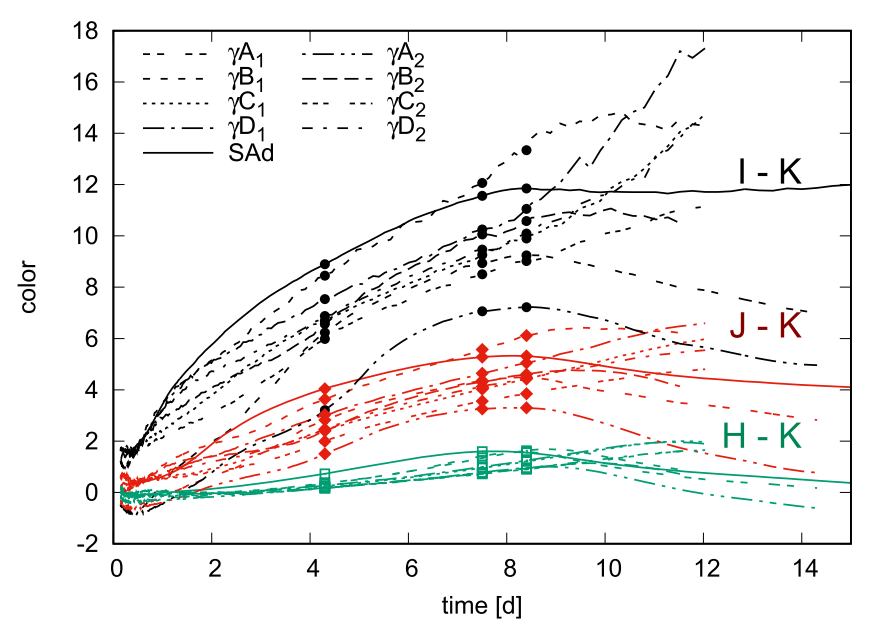

Figure 1. $I-K$ color (black circles), $J-K$ color (red diamonds), and $H-K$ color (green squares) evolution as a function of time since the neutron star merger. Even a shallow infrared search for kilonovae would be more constraining than an optical search. $\mathrm{H}$ band may be the most optimal ground-based infrared filter given the relatively lower sky brightness and small $H-K$ colors.

\section{Discussion}

First, we investigate whether our Ks-band detection of GRB $160821 \mathrm{~B}$ in the first epoch at 4.3 days is consistent with afterglow emission from the jet. If we extrapolate $\mathrm{V}$ mag of the afterglow reported in Troja et al. (2016) at 3.6 days, and assume a power-law decay based on fitting to afterglow photometry reported in $\mathrm{Xu}$ et al. (2016), we find that the $V-K$ color is approximately $1.9 \mathrm{mag} \mathrm{AB}$. This $V-K$ color is somewhat redder than that expected from a typical SHB afterglow spectral index but it can possibly be explained by dust. Looking up the extensive compilation of afterglow data of short hard bursts by Fong et al. (2015), we find that there is remarkably little Ks-band data that we can directly compare to (note that the latest phase of a near-IR afterglow detection in this compilation is only 1.5 days). The $V-K$ color is inconsistent with all the kilonova models presented in this Letter that predict colors redder than 6 mag at this phase (Figure 1). The $V-K$ color is also not as red as models presented in Barnes et al. (2016) or Rosswog et al. (2016) (see Figure 2). Furthermore, our Ks-band non-detections in the second two epochs at 7.5 days and 8.7 days are also consistent with the hypothesis that there was no detectable kilonova emission from this GRB (see Figure 2). Additional contemporaneous multi-band multi-epoch photometry is necessary to securely disentangle whether or not there could be a contribution from both an afterglow and a kilonova at this 4.3 day epoch. For example, if there was evidence to rule out a dust contribution, the excess emission could perhaps be explained by relatively bluer kilonova models with different assumptions on ejecta composition and ejecta opacity.

Proceeding despite this caveat, we applied the empirical scaling laws discussed in Section 3 to rescale the Ks-band light 


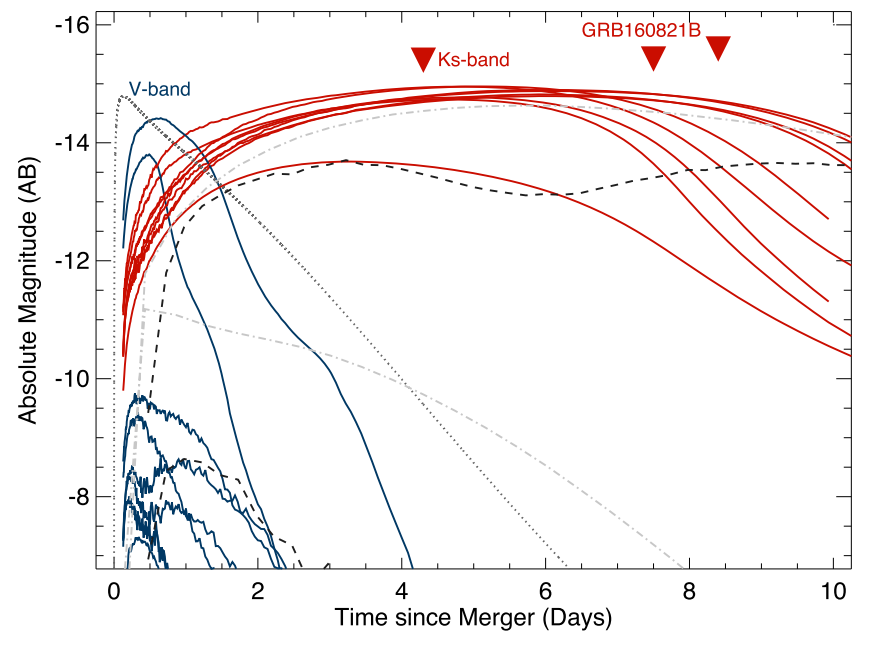

Figure 2. Comparing the GRB 160821B upper limits to model predictions in the Ks band (red solid lines) and $\mathrm{V}$ band (blue solid lines) assuming an ejecta mass of $0.01 M_{\odot}$ and ejecta velocity of $0.1 \mathrm{c}$. Also shown for comparison are other kilonova models in the literature with similar ejecta mass and ejecta velocity assumptions by Barnes et al. (2016; black dashed line, Ks band, and $\mathrm{V}$ band) and Rosswog et al. (2016; light gray dashed-dotted line, Ks band and V band, "ns12n14-dz31" model). The bluest models are powered by beta decay of free neutrons (gray dotted line; Metzger et al. 2015).

curves for each of our models to a different value of mass and velocity, for a range of masses and velocities. We then sampled the resulting redshifted light curves at the observed epochs and marked out regions with magnitudes excluded by upper limits at 4.3 days and 7.5 days. The result is shown in Figure 3, where we plot excluded area for each model in the mass-velocity parameter space. The upper limit at epoch 8.7 days does not provide additional constraints, so we do not show corresponding areas. Given that most simulations predict ejecta velocities higher than $0.1 \mathrm{c}$ (Hotokezaka et al. 2013; Rosswog 2013), we conclude that the mass of the dynamical ejecta of GRB 160821B is less than $0.03 M_{\odot}$. We note that this constraint is broadly consistent with other less red models in the literature (Barnes et al. 2016; Rosswog et al. 2016) within a factor of few (see Figure 2).

To further characterize the parameter space of this family of models, we look at the predicted model emission as a function of ejecta mass and ejecta velocity using the empirical scaling laws described above (see Figure 4). Future gravitational-wave detections of neutron star mergers will be relatively nearby due to the sensitivity of advanced gravitational-wave interferometers being limited to approximately $200 \mathrm{Mpc}$ (Abbott et al. 2016). At this distance limit, similar ground-based Ks-band photometry would be extremely constraining for all models including the faintest SAd model with dynamical ejecta only (Figure 4) for any assumption in ejecta mass or velocity spanning two orders of magnitude. For comparison in Figure 4, we also show the J-band detection of GRB 130603B $(-15.35$ mag at 7 days in the comoving frame; Tanvir et al. 2013). The models presented here would suggest a very high ejecta mass, $m_{\mathrm{ej}}>0.08 M_{\odot}$ (or extreme velocities $>0.4 c$, which also shifts the peak to earlier time). However, one caveat here is that the nuclear mass model FRDM tends to underestimate nuclear heating rates in comparison with other mass models (Duflo \& Zuker 1995), and hence produces dimmer kilonovae (Rosswog et al. 2016).

Examining the extremely red color evolution further (Figure 1), we find $I-K$ s colors peaking between $7-16$ mag.

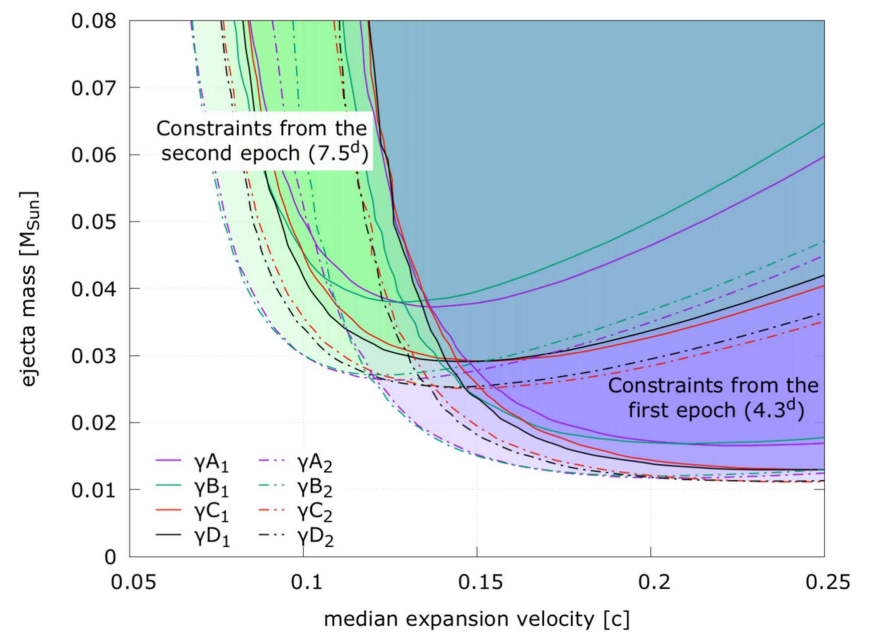

Figure 3. Ks-band upper limit for GRB $160821 \mathrm{~B}$ at 4.3 days and 7.5 days rules out the purple shaded and green shaded regions, respectively, with higher ejecta mass and higher ejecta velocity than denoted by the contours above. Each contour represents one of the eight models for each of two epochs. Assuming model predictions of dynamical ejecta in excess of $0.1 \mathrm{c}$, our observations suggest ejecta masses lower than $0.03 M_{\odot}$ for this event.

This suggests that even a relatively shallow infrared search for a kilonova would be competitive and complementary to constraints from optical searches (e.g., Kasliwal et al. 2016; Smartt et al. 2016; Soares-Santos et al. 2016). The H-Ks color is relatively small, suggesting that either filter would work well. Space-based observations, unhindered by night sky brightness, are deeper but are currently limited to the $\mathrm{H}$ band with narrow field-of-view cameras on board the Hubble Space Telescope (until the launch of the James Webb Space Telescope and Wide Field Infrared Survey Telescope).

Infrared searches for kilonovae associated with coarsely localized gravitational-wave triggers are currently inhibited by the astronomical cost of wide-field infrared detectors. The best effort currently is the $0.6 \mathrm{deg}^{2}$ field-of-view camera on the $4 \mathrm{~m}$ VISTA telescope (Sutherland et al. 2015). For example, VISTA covered $8 \%$ of the localization of GW150914 to a depth of $J<20.7$ (Abbott et al. 2016; GCN \#18353). While the depth is constraining (corresponds to -14.3 at $100 \mathrm{Mpc}$ ), the fractional area covered is too small. A future wide-field survey, say in the $\mathrm{H}$ band or Ks band to a similar depth, but covering a larger fraction of the error circle would be constraining in this context. We are exploring alternative semiconductors (Sullivan et al. 2014; R. Simcoe et al. 2017, in preparation) and/or creative optical design at a polar location (Moore et al. 2016) to break this cost barrier and/or blinding night-sky barrier to explore the dynamic infrared sky.

M.M.K. thanks M. Heida, F. Fuerst, and E. S. Phinney for cooperating with the Target Of Opportunity interrupt observations at Keck I. We thank S. B. Cenko, J. Barnes, D. Kasen, R. Simcoe, D. A. Perley, S. R. Kulkarni, W. Fong, N. Lloyd-Ronning, and W. Even for valuable discussions. We thank our anonymous referee for helpful feedback. This work was supported by the GROWTH (Global Relay of Observatories Watching Transients Happen) project funded by the National Science Foundation Partnership in International Research Program under NSF PIRE grant number 1545949. Work at LANL was done under the auspices of the National Nuclear Security Administration of the U.S. Department of 

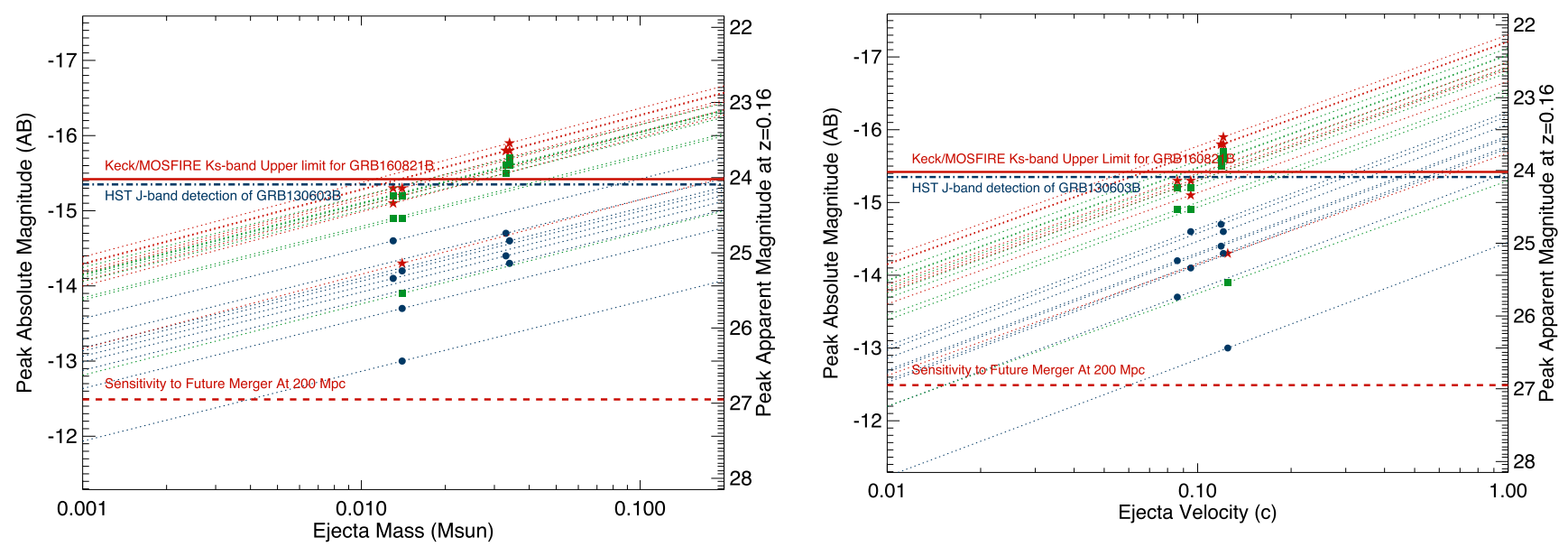

Figure 4. Theoretical model predictions for the $\mathrm{J}$ band (blue circle), $\mathrm{H}$ band (green square), and Ks band (red star) as a function of ejecta mass (left panel) and ejecta velocity (right panel). Dotted lines show a rough empirical scaling with ejecta mass (left panel) and ejecta velocity (right panel). Our current Ks-band upper limit for GRB 160821B constrains high ejecta mass and high ejecta velocity (solid red line). A future gravitational-wave detection (dashed red line), at say $200 \mathrm{Mpc}$, would be so nearby that the same data would be able to test all nine models covering the entire parameter space. Previously reported J-band detections of GRB 130603B (dashed blue line) cannot be easily explained by the models studied in this Letter.

Energy at Los Alamos National Laboratory under Contract No. DE-AC52-06NA25396. All LANL calculations were performed on LANL Institutional Computing resources.

Facility: Keck:I (MOSFIRE).

\section{References}

Abbott, B. P., Abbott, R., Abbott, T. D., et al. 2016, ApJL, 826, L13

Abbott, B. P., Abbott, R., Abbott, T. D., et al. 2016, LRR, 19, 1

Arcones, A., \& Martínez-Pinedo, G. 2011, PhRvC, 83, 045809

Barnes, J., \& Kasen, D. 2013, ApJ, 775, 18

Barnes, J., Kasen, D., Wu, M.-R., \& Martínez-Pinedo, G. 2016, ApJ, 829, 110

Berger, E. 2014, ARA\&A, 52, 43

Berger, E., Fong, W., \& Chornock, R. 2013, ApJL, 774, L23

Duflo, J., \& Zuker, A. P. 1995, PhRvC, 52, R23

Fernández, R., \& Metzger, B. D. 2016, ARNPS, 66, 23

Fong, W., Alexander, K. D., \& Laskar, T. 2016, GCN, 19854, 1

Fong, W., Berger, E., Margutti, R., \& Zauderer, B. A. 2015, ApJ, 815, 102

Fong, W., Berger, E., Metzger, B. D., et al. 2014, ApJ, 780, 118

Fong, W., Margutti, R., Chornock, R., et al. 2016, ApJ, 833, 151

Fontes, C. J., Fryer, C. L., Hungerford, A. L., et al. 2017, arXiv:1702.02990

Galama, T. J., Vreeswijk, P. M., van Paradijs, J., et al. 1998, Natur, 395, 670

Gehrels, N., Chincarini, G., Giommi, P., et al. 2004, ApJ, 611, 1005

Grossman, D., Korobkin, O., Rosswog, S., \& Piran, T. 2014, MNRAS, 439, 757

Hotokezaka, K., Kiuchi, K., Kyutoku, K., et al. 2013, PhRvD, 87, 024001

Jin, Z.-P., Hotokezaka, K., Li, X., et al. 2016, NatCo, 7, 12898

Jin, Z.-P., Li, X., Cano, Z., et al. 2015, ApJL, 811, L22

Kasen, D., Badnell, N. R., \& Barnes, J. 2013, ApJ, 774, 25

Kasen, D., Fernández, R., \& Metzger, B. D. 2015, MNRAS, 450, 1777

Kasliwal, M. M., Cenko, S. B., Singer, L. P., et al. 2016, ApJL, 824, L24

Korobkin, O., Rosswog, S., Arcones, A., \& Winteler, C. 2012, MNRAS, 426, 1940
Levan, A. J., Wiersema, K., Tanvir, N. R., et al. 2016, GCN, 19846, 1 Li, L.-X., \& Paczyński, B. 1998, ApJL, 507, L59

Lü, H.-J., Zhang, H.-M., Zhong, S.-Q., et al. 2017, ApJ, 835, 181

McLean, I. S., Steidel, C. C., Epps, H. W., et al. 2012, Proc. SPIE, 8446, 84460J Metzger, B. D., Bauswein, A., Goriely, S., \& Kasen, D. 2015, MNRAS, 446, 1115

Möller, P., Nix, J. R., Myers, W. D., \& Swiatecki, W. J. 1995, ADNDT, 59, 185 Mooley, K. P., Staley, T. D., Fender, R. P., et al. 2016, GCN, 19898, 1

Moore, A. M., Kasliwal, M. K., Gelino, C. R., et al. 2016, Proc. SPIE, 9906, 99062C

Panov, I. V., Kolbe, E., Pfeiffer, B., et al. 2005, NuPhA, 747, 633

Panov, I. V., Korneev, I. Y., Rauscher, T., et al. 2010, A\&A, 513, A61

Perego, A., Rosswog, S., Cabezón, R. M., et al. 2014, MNRAS, 443, 3134

Perley, D. A., Metzger, B. D., Granot, J., et al. 2009, ApJ, 696, 1871

Rauscher, T., \& Thielemann, F.-K. 2000, ADNDT, 75, 1

Rosswog, S. 2013, RSPTA, 371, 20120272

Rosswog, S., Feindt, U., Korobkin, O., et al. 2016, arXiv:1611.09822

Rosswog, S., Korobkin, O., Arcones, A., Thielemann, F.-K., \& Piran, T. 2014, MNRAS, 439, 744

Siegel, M. H., Barthelmy, S. D., Burrows, D. N., et al. 2016, GCN, 19833, 1 Skrutskie, M. F., Cutri, R. M., Stiening, R., et al. 2006, AJ, 131, 1163

Smartt, S. J., Chambers, K. C., Smith, K. W., et al. 2016, MNRAS, 462, 4094 Soares-Santos, M., Kessler, R., Berger, E., et al. 2016, ApJL, 823, L33

Sullivan, P. W., Croll, B., \& Simcoe, R. A. 2014, Proc. SPIE, 9154, 91541F

Sutherland, W., Emerson, J., Dalton, G., et al. 2015, A\&A, 575, A25

Tanvir, N. R., Levan, A. J., Fruchter, A. S., et al. 2013, Natur, 500, 547

Thielemann, F.-K., Arcones, A., Käppeli, R., et al. 2011, PrPNP, 66, 346

Troja, E., Tanvir, N., Cenko, S. B., et al. 2016, GCN, 20222, 1

Winteler, C. 2014, PhD thesis, Univ. Basel

Wollaeger, R. T., Korobkin, O., Fontes, C. J., et al. 2017, arXiv:1705.07084

Wollaeger, R. T., \& van Rossum, D. R. 2014, ApJS, 214, 28

Xu, D., Malesani, D., de Ugarte Postigo, A., Gafton, E., \& Rivero Losada, I. 2016, GCN, 19834, 1 